\title{
LOS PRIMEROS DIENTES DE ANQUILOSAURIO (ORNITHISCHIA: THYREOPHORA) DESCRITOS EN EL CRETÁCICO INFERIOR DE ESPAÑA
}

\author{
José Ignacio CANUDO, José Ignacio RUIZ- \\ OMEÑACA y Gloria CUENCA-BESCÓS
}

\author{
Área y Museo de Paleontología. Facultad de Ciencias. \\ Universidad de Zaragoza. 50009 Zaragoza \\ jicanudo@unizar.es,jigruiz@unizar.es, cuencag@unizar.es
}

Canudo, J.I., Ruiz-Omeñaca, J. I. y Cuenca-Bescós, G. 2004. Los primeros dientes de Anquilosaurio (Ornithischia: Thyreophora) descritos en el Cretácico Inferior de España. [The first Ankylosaurian teeth (Ornithischia: Thyreophora) described from Lower Cretaceous in Spain.] Revista Española de Paleontología, 19 (1), $33-46$. ISSN 0213-6937.

\begin{abstract}
The La Cantalera locality has yielded the first Lower Cretaceous ankylosaurian teeth from Spain. The site is located at the Blesa Formation (Josa, Teruel). The charophyte assemblage gives an uppermost Hauterivianlowermost Barremian age to the site. The smooth enamel and the absence of cingulum are characteristic of these teeth. The current phylogenetic proposals consider the lack of cingulum primitive for Ankylosauria, however in cladistic analysis of different authors, outgroups considered for Ankylosauria do have cingulum. For these reasons we consider primitive the presence of cingulum in Ankylosauria, and thus, the lack of cingulum the derived condition. The absence of cingulum is shared between La Cantalera teeth and Gastonia, a polacanthid from the Barremian of USA. Therefore we classify La Cantalera Ankylosauria teeth as a Polacanthidae indet.
\end{abstract}

Keywords: Lower Cretaceous, Teruel, Ankylosauria, Polacanthidae, teeth.

\section{RESUMEN}

Se describen por primera vez en España, dos dientes de un Ankylosauria del Cretácico Inferior. El material proviene de la Formación Blesa en La Cantalera (Josa, Teruel), datada con carofitas como Hauteriviense terminal-Barremiense basal. Sus caracteres diagnósticos son el esmalte liso y una base inflada, sin cíngulo. En las propuestas filogenéticas de Ankylosauria la ausencia de cíngulo se considera como el estadio primitivo para este carácter. Sin embargo los grupos hermanos considerados en estas propuestas tienen un cíngulo desarrollado. Por tanto consideramos que en Ankylosauria la presencia de cíngulo es el estadio primitivo, y la ausencia es el derivado. Los dientes de La Cantalera comparten la ausencia de cíngulo con el polacántido Gastonia (Barremiense de EE.UU.), por lo que hemos clasificado al Ankylosauria de La Cantalera como Polacanthidae indet.

Palabras clave: Cretácico Inferior, Teruel, Ankylosauria, Polacanthidae, dientes.

\section{INTRODUCCIÓN}

Los anquilosaurios son un grupo de dinosaurios ornitisquios, cuadrúpedos y con el cuerpo cubierto de placas dérmicas (Coombs, 1978). En la actualidad se incluyen dentro de Thyreophora junto a Stegosauria y otros taxones relacionados (Sereno, 1999). Tradicionalmente Ankylosauria se ha separado en dos familias: Ankylosau- ridae y Nodosauridae al poseer los anquilosáuridos una porra (bone club) al final de la cola (Coombs, 1978). En la actualidad se diferencian por más caracteres, especialmente del cráneo (Coombs y Maryanska, 1990). El esquema clásico está cambiando, al reconocerse una tercera familia (Polacanthidae) con caracteres que se consideraban diagnósticos para una u otra de estas dos familias (Carpenter, 2001). 
Los restos fósiles de Ankylosauria son relativamente frecuentes en Laurasia a partir del Aptiense (Coombs y Maryanska, 1990; Carpenter y Kirkland, 1998). Se han descrito cinco o más géneros en cada periodo geológico con excepción del Coniaciense (McCrea et al., 2001). Sin embargo hay pocos taxones descritos en la parte baja del Cretácico, y suelen estar representados por material fragmentario; la única excepción es un ejemplar juvenil completo de la Formación Yixian en China (Xu et al., 2001) recientemente descubierto. Posiblemente, este pobre registro se debe a la escasez de yacimientos con dinosaurios, y no a la rareza de estos ornitisquios en los ecosistemas continentales del Cretácico Inferior.

Una de las áreas en Europa más importantes para el estudio de los dinosaurios del Cretácico Inferior es la Cordillera Ibérica. En ella afloran sedimentos continentales y de transición que contienen restos de vertebrados continentales situados en secuencias estratigráficas potentes y continuas que están permitiendo conocer la evolución paleoambiental de los ecosistemas del Cretácico Inferior de esta parte de Europa (Díaz-Molina et al., 1985; Sanz et al., 1987; Ruiz-Omeñaca et al., 1997; Canudo et $a l ., 1997)$. En el marco del proyecto VECOBA (VErtebrados COntinentales del BArremiense) se ha comenza- do la excavación y estudio de La Cantalera, uno de los yacimientos más prometedores de la Cordillera Ibérica debido a su gran superficie de exposición y a la excepcional abundancia y diversidad de restos desarticulados de vertebrados, especialmente dinosaurios (Ruiz-Omeñaca et al., 1997; Ruiz-Omeñaca y Canudo, 2001; Canudo et al., 2002a, b). En este trabajo se describen dos dientes de anquilosaurio de La Cantalera. Además de ser los primeros descritos en el Cretácico Inferior de España, permiten mostrar la presencia de este grupo de dinosaurios en niveles del tránsito Hauteriviense-Barremiense de la Cordillera Ibérica, ampliando su distribución geográfica.

\section{Los Anquilosaurios del Cretácico Inferior en España}

Los restos de anquilosaurios del Cretácico Inferior de España (Tabla 1) son escasos y fragmentarios (Pereda Suberbiola y Galton, 2001). Todos los yacimientos se localizan en la Cordillera Ibérica: Cuenca de Cameros Occidental (Sector Norte en la provincia de Burgos; Sector de Soria en la provincia de Soria) y Cuenca del Maestrazgo (Subcuencas de Oliete y Galve en la provincia de Teruel; Subcuenca de Morella en la provincia de Castellón).

La provincia donde se encuentran más restos de Ankylosaria publicados es Burgos. Los materiales más

\begin{tabular}{|l|l|l|l|}
\hline $\begin{array}{l}\text { Yacimiento (localidad, } \\
\text { provincia) / Locality }\end{array}$ & $\begin{array}{l}\text { Edad (Formación) / Age } \\
\text { (Formation) }\end{array}$ & Clasificación / assignment & Referencia / Reference \\
\hline $\begin{array}{l}\text { Mas Romeu (Morella, } \\
\text { Castellón) }\end{array}$ & Aptiense inferior (Morella) & ? Nodosauridae indet. & Santafé et al., 1982 \\
\hline $\begin{array}{l}\text { Mas de la Parreta, CMP-3 } \\
\text { (Morella, Castellón) }\end{array}$ & Aptiense inferior (Morella) & Polacanthus sp. & Gasulla et al., 2003 \\
\hline $\begin{array}{l}\text { Fuente Espudia (Salas de los } \\
\text { Infantes, Burgos) }\end{array}$ & $\begin{array}{l}\text { Hauteriviense superior- } \\
\text { Barremiense inferior (Pinilla } \\
\text { de los Moros) }\end{array}$ & Polacanthus sp. & $\begin{array}{l}\text { Pereda Suberbiola } \text { et al., } \\
1999\end{array}$ \\
\hline $\begin{array}{l}\text { Los Peñucos-La Ballesta } \\
\text { (Salas de los Infantes, } \\
\text { Burgos) }\end{array}$ & $\begin{array}{l}\text { Barremiense superior- } \\
\text { Aptiense (Castrillo de la } \\
\text { Reina) }\end{array}$ & cf. Polacanthus sp. & $\begin{array}{l}\text { Pereda Suberbiola } \text { et al., } \\
1999\end{array}$ \\
\hline $\begin{array}{l}\text { ANK-Barranco (Galve, } \\
\text { Teruel) }\end{array}$ & $\begin{array}{l}\text { Barremiense inferior } \\
\text { (Camarillas) }\end{array}$ & Ankylosauria indet. & $\begin{array}{l}\text { Ruiz-Omeñaca y Canudo, en } \\
\text { prensa (sin describir/ } \\
\text { undescribed) }\end{array}$ \\
\hline $\begin{array}{l}\text { Tenada Tizuela (Aldea del } \\
\text { Pinar, Burgos) }\end{array}$ & $\begin{array}{l}\text { Hauteriviense superior- } \\
\text { Barremiense inferior } \\
\text { (Piedrahita de Muñó) }\end{array}$ & Nodosauridae indet. & $\begin{array}{l}\text { Pereda Suberbiola } \text { } \text { et al., } \\
\text { 2003 (sin describir/ } \\
\text { undescribed) }\end{array}$ \\
\hline Zorralbo (Golmayo, Soria) & $\begin{array}{l}\text { Hauteriviense terminal- } \\
\text { Barremiense basal (Golmayo) }\end{array}$ & Nodosauridae indet. & $\begin{array}{l}\text { Fuentes-Vidarte } \text { et al., 2003 } \\
\text { (sin describir/ undescribed) }\end{array}$ \\
\hline La Cantalera (Josa, Teruel) & $\begin{array}{l}\text { Hauteriviense superior- } \\
\text { Barremiense basal (Blesa) }\end{array}$ & Polacanthidae indet. & este trabajo/ this paper \\
\hline
\end{tabular}

Tabla 1. Distribución de los anquilosaurios en el Cretácico Inferior de España. Sólo se menciona la referencia en la que aparece la asignación más actual; para otras referencias véase el texto.

Distribution of Lower Cretaceous ankylosaurs in Spain. Only the last published references are cited; for older ones see the text. 
antiguos provienen de Tenada Tizuela (Aldea del Pinar), donde se han encontrado vértebras caudales sin describir de un anquilosaurio, citadas como nodosáurido indeterminado por Pereda Suberbiola y Galton (2001) y como anquilosaurio por Pereda Suberbiola et al. (2003). El yacimiento se sitúa en la Formación Piedrahita de Muñó (Grupo Tera), equivalente a la Formación Pinilla de los Moros (Pereda Suberbiola et al., 2003), de edad Hauteriviense superior-Barremiense inferior (MartínClosas y Alonso-Millán, 1998).

Sanz (1983) y Sanz et al. (1990, 1992) en la facies Weald de Salas de los Infantes (yacimiento de Los Peñucos-La Ballesta) describen y figuran una espina dérmica. La posición estratigráfica de este yacimiento varía según los autores: Barremiense-Albiense (Sanz, 1983), Hauteriviense (Torcida Fernández-Baldor, 1996); Hauteriviense-Aptiense (Pereda Suberbiola et al., 1999). Dado que se encuentra en la Formación Castrillo de la Reina (Torcida Fernández-Baldor et al., en prensa) su edad con carofitas es Barremiense superior-Aptiense (MartínClosas y Alonso-Millán, 1998). Esta espina ha tenido distintas atribuciones tanto anatómicas, como taxonómicas. Anatómicamente fue en principio interpretada como espina presacra (Sanz, 1983), posteriormente como espina dorsolateral (Sanz et al., 1990), que no concuerda ni con la posición que se observa en la figura 31 de estos mimos autores, ni con la interpretación de otros autores como Pereda-Suberbiola et al. (1999) que la sitúan en una posición cervical (al igual que la figura 31 de Sanz et al., 1990). Inicialmente, Sanz (1983) la asigna a Hylaeosaurus teniendo en cuenta su supuesta sinonimia con Polacanthus. La publicación de Coombs (1978) influyó mucho en la sistemática de los trabajos contemporáneos en su propuesta de sinonimia entre Polacanthus e Hylaeosaurus. Sin embargo, posteriormente se ha comprobado que estos dos géneros provienen de niveles estratigráficos distintos y tienen suficientes caracteres para poderlos dife- renciar; por lo que definitivamente Pereda Suberbiola et al. (1999) la asignan a Polacanthus sp. También en la formación Castrillo de la Reina en Salas de los Infantes (yacimiento de Fuente Espudia), Pereda Suberbiola et al. (1999) describen dos espinas caudales anteriores, un osteodermo y varios fragmentos de costillas, posiblemente un único individuo clasificado como Polacanthus sp.

También en Cameros Occidental se encuentra el yacimiento de Zorralbo (Golmayo, Soria), con restos de un aquilosaurio, por el momento sin describir (Ankylosauria indet. en Fuentes Vidarte et al., 2002; Nodosauridae indet. en Fuentes Vidarte et al., 2003). Estos restos se encuentran en la Formación Golmayo, de edad Hauteriviense terminal-Barremiense basal según Martín-Closas y Alonso-Millán (1998). Futuros estudios clarificarán la posición sistemática del anquilosaurio de Golmayo.

En la Cuenca del Maestrazgo (subcuenca de Morella) se hallan los restos de anquilosaurios más modernos del Cretácico Inferior español, todos procedentes de la Formación Morella (Aptiense inferior): un osteodermo y una falange de ?Nodosauridae indet. (Santafé et al., 1982) asignados a un nodosaurio por la morfología del osteodermo y por la ausencia de Ankylosauridae en Europa cuando se describieron) y un fragmento de escudo sacral, un osículo y dos espinas dérmicas atribuidos a Polacanthus (Gasulla et al., 2003).

En la Subcuenca de Galve (Galve, Teruel), Canudo et al. (1997) citan una espina presacra en la parte media de la Formación Camarillas (Barremiense inferior, DíazMolina et al., 1985). Aunque no se ha descrito, se ha citado como Nodosauridae indet. (Canudo et al., 1996; Pereda Suberbiola y Galton, 2001) y como Ankylosauria indet. (Ruiz-Omeñaca y Canudo, en prensa). También en la provincia de Teruel se han citado restos de anquilosaurio en La Cantalera (Josa, Teruel). Son dientes, osteodermos, costillas y una vértebra caudal de anquilosaurio que inicialmente se citaron como Nodosauridae indet. en Ruiz-

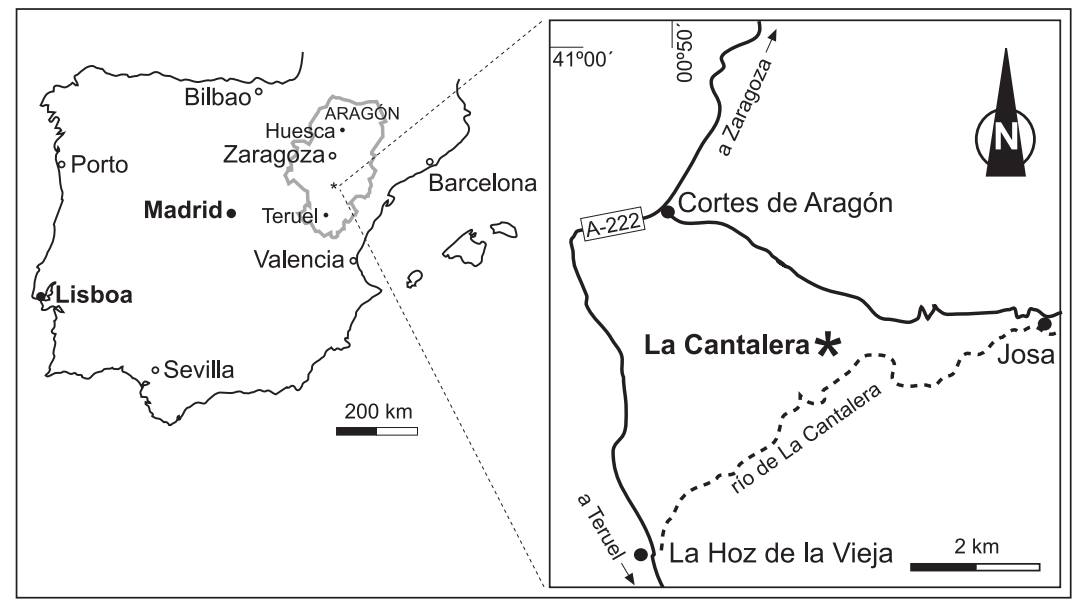

Figura 1. Situación geográfica del yacimiento de la Cantalera, tránsito Hauteriviense-Barremiense (Josa, Teruel). Geographic map of La Cantalera site (Josa, Teruel, Spain), Hauterivian-Barremian transition. 
Omeñaca y Canudo (2001) y como Ankylosauria indet. en Canudo et al. (2002a) y Ruiz-Omeñaca y Canudo (en prensa). Precisamente dos dientes de este yacimiento son los que se estudian en este trabajo.

Resumiendo, los anquilosaurios del Cretácico Inferior español están representados por material fragmentario, y por tanto poco diagnóstico: vértebras caudales, osteodermo y falange (Nodosauridae indet.), una espina presacra (Ankylosauria indet.) y una espina cervical (cf. Polacanthus sp.). La única excepción son dos espinas caudales, un osteodermo y varios fragmentos de costillas de Polacanthus sp. en Salas de los Infantes, y varios elementos de la armadura dérmica de Polacanthus sp. en Morella.

\section{SITUACIÓN GEOLÓGICA Y GEOGRÁFICA}

Geológicamente, La Cantalera se encuentra en la Subcuenca de Oliete, y geográficamente cerca de la localidad de Josa, Teruel (Fig. 1). El yacimiento se sitúa en unas arcillas grises lacustres-palustres depositadas cerca de la base de la Formación Blesa (Ruiz-Omeñaca et al., 1997; Canudo et al., 2002a). Entre los microfósiles (recuperados mediante técnicas de lavado-tamizado) son abundantes los ostrácodos y las carófitas, que forman la mayor parte del residuo del tamiz por encima de 50 micras. Se han reconocido ostrácodos frecuentes en los sedimentos lacustres y salobres del Hauteriviense superior y Barremiense del Norte de España (Ruiz-Omeñaca et al., 1997). Las carófitas están representadas por utrículos de clavatoraceas y abundantes talos. La asociación es bioestratigráficamente similar a la que se encuentra en el techo de la Fm. El Castellar de la subcuenca de Galve (que junto con la Subcuenca de Las Parras formaba hasta 1996 la llamada Cuenca de Aliaga, véase Soria de Miguel, 1997), datado como Hauteriviense superior-Barremiense basal (Martín-Closas, 2000; Canudo et al., 2002a).

Los restos directos e indirectos de vertebrados son frecuentes en La Cantalera (Tabla 2). Entre los restos indirectos hay pequeños fragmentos de cáscara de huevo de tortugas, cocodrilos y dinosaurios, posiblemente de terópodos. Se han reconocido coprolitos de pequeño tamaño, al menos de nueve morfologías distintas. Los restos directos más abundantes son dientes aislados, siendo los más frecuentes los de cocodrilos. También hay restos postcraneales desarticulados, y excepcionalmente se han encontrado vértebras caudales articuladas. Entre los microvertebrados se han identificado dientes de mamíferos multituberculados albionbatáridos, placas periferales de pequeño tamaño pertenecientes a una tortuga criptodira de la familia Pleurosternidae?, y dientes aislados de al menos un Pterosauria indet.

Los dinosaurios son los vertebrados más diversos; están representados por dientes y restos postcraneales de saurópodos, terópodos, ornitópodos y tireóforos. Los saurópodos son escasos, habiéndose identificado Euhelopodidae indet., una familia que se encuentra bien diversificada en el Cretácico Inferior de Asia (Canudo et al., 2002b). Los dientes de terópodos son relativamente abundantes. Al menos están representados cuatro taxones distintos: Theropoda indet., Baryonychinae indet., «Prodeinodon»sp. y dientes de «tipo Dromaeosauridae»

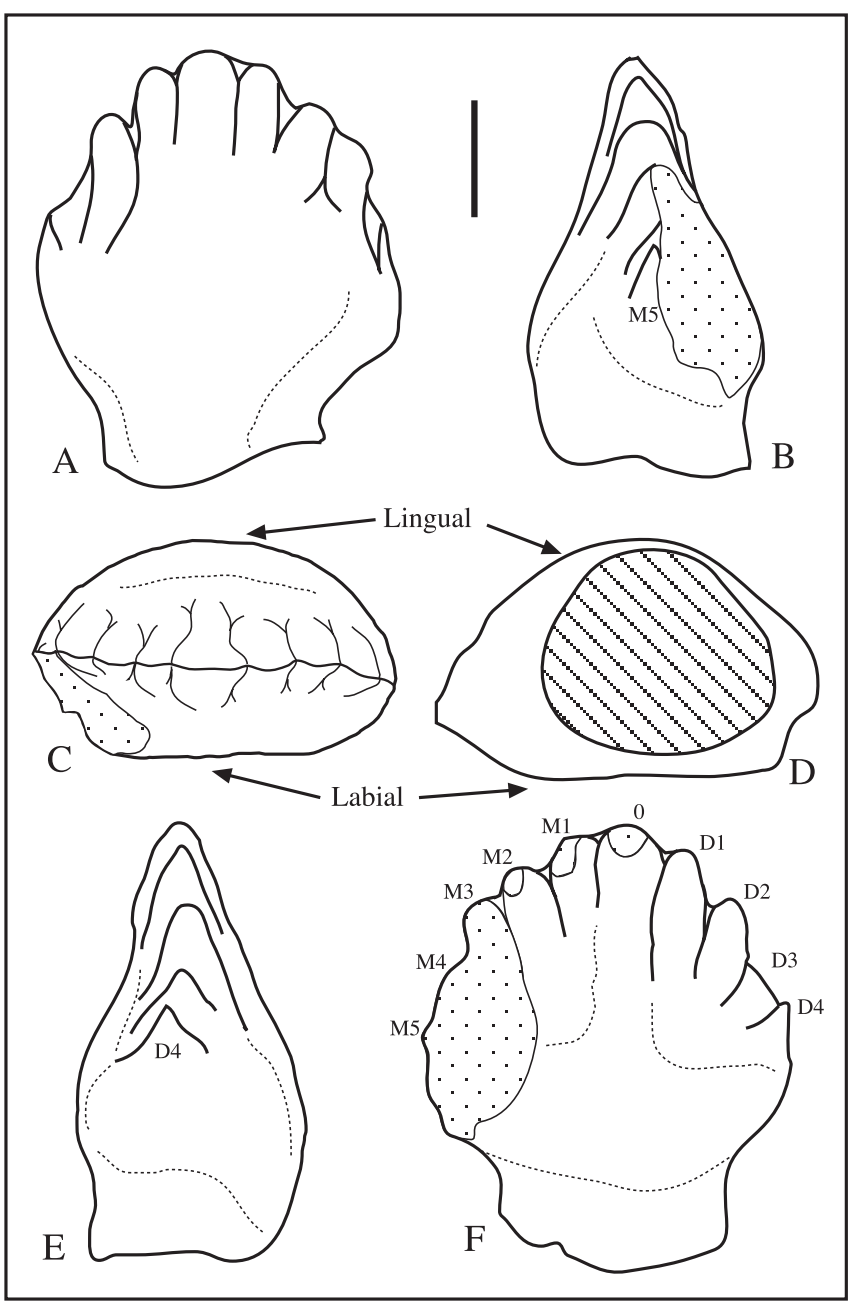

Figura 2. MPZ 2002/926. Dibujos esquemáticos del diente mandibular izquierdo del polacántido de La Cantalera, en vistas lingual (A), mesial (B), oclusal (C), adapical (D), distal (E) y labial (F). En F se presenta la nueva terminología para denominar los dentículos de Ankylosauria: 0 es el dentículo central, D son los dentículos distales y M los mesiales. El punteado representa las facetas de desgaste y el rayado la rotura de la raíz. La escala es $1 \mathrm{~mm}$.

MPZ 2002/926. Drawings of the left dentary tooth of the La Cantalera polacanthid, in lingual (A), mesial (B), occlusal (C), adapical, (D) distal, $(E)$ and labial $(F)$ views. In $F$ there is the new terminology for ankylosaurian teeth: 0 , apical denticle; $D$, distal denticles; $M$, mesial denticles. Doted areas represent the wear facets, lines represent the broken part of the root. Scale bar $=1 \mathrm{~mm}$. 


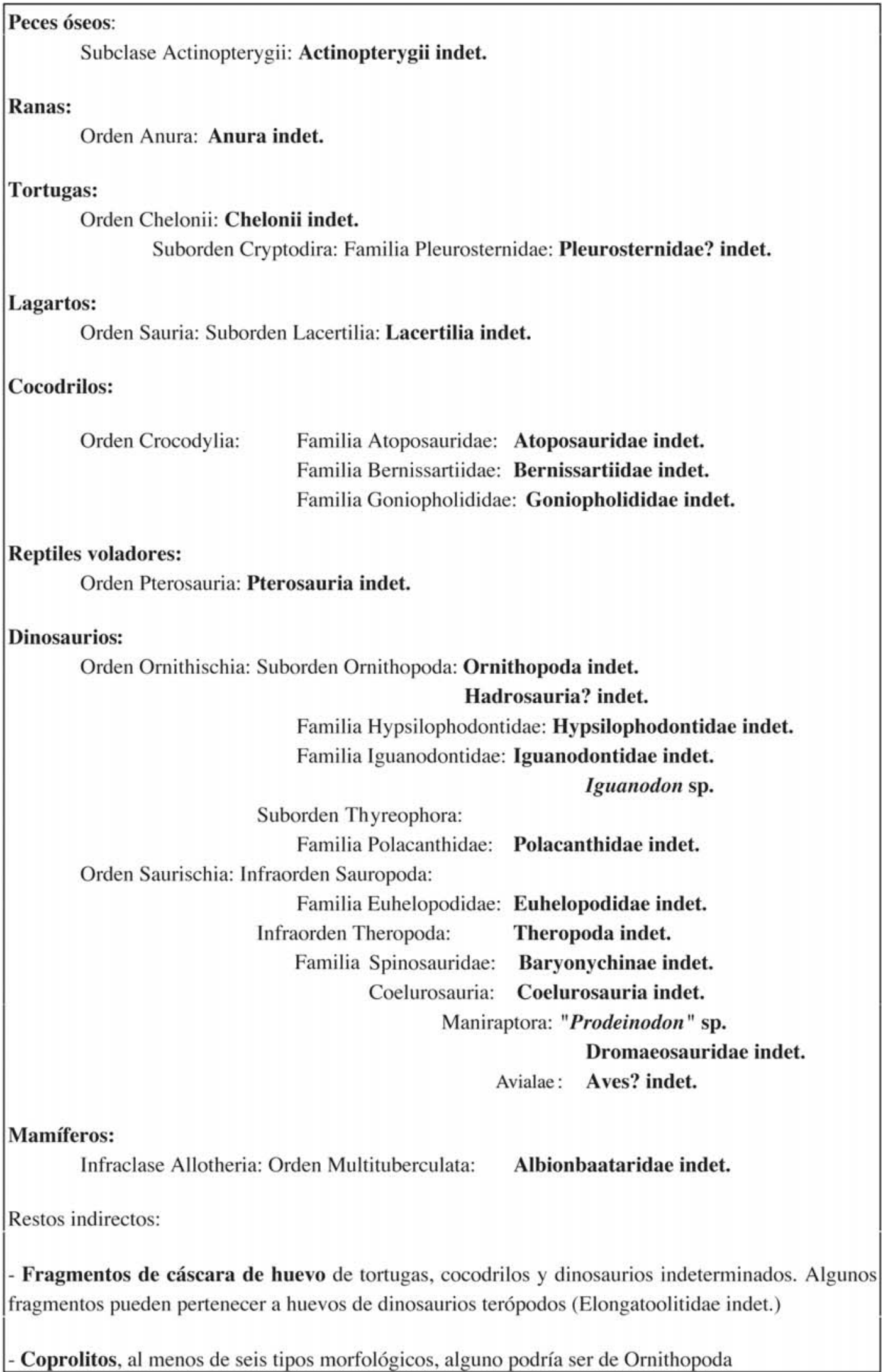

Tabla 2. Lista faunística de los vertebrados de La Cantalera, tránsito Hauteriviense-Barremiense (Josa, Teruel). Ver referencias en Ruiz-Omeñaca y Canudo (2001) y Canudo et al. (2002b).

Vertebrate faunal list of La Cantalera (Josa, Teruel, Spain), Hauterivian-Barremian transition. Modified from RuizOmeñaca \& Canudo (2001) and Canudo et al. (2002b). 
(Ruiz-Omeñaca y Canudo, 2001; Ruiz-Omeñaca y Canudo, 2003). Los restos de dinosaurio más abundantes son los dientes mudados de ornitópodos. Al menos hay tres taxones representados: Hypsilophodontidae indet., «Iguanodontidae» indet. e Iguanodon sp. (Ruiz-Omeñaca et al., 1997). Además se han encontrado dientes sin crestas secundarias asignados a Hadrosauria? indet. (RuizOmeñaca y Canudo, 2001). Los anquilosaurios están representados por placas dérmicas, costillas y una vértebra caudal, en periodo de preparación, y los dos dientes objeto de este estudio.

\section{MATERIAL Y MÉTODOS}

Los dos dientes que se estudian en este trabajo se han recuperado en el concentrado obtenido por el lavado-tamizado del sedimento de la excavación, disgregado con agua caliente y agua oxigenada al $10 \%$ aproximadamente. Se ha tamizado con un tamiz de $50 \mu \mathrm{m}$, separándose todos los restos reconocibles de vertebrados. El material estudiado proviene del lavado de unos $1100 \mathrm{~kg}$ de las campañas de excavación de 2000 y 2002. La nomenclatura utilizada para describir los dientes sigue las propuestas más comunes (Coombs y Maryanska, 1990; Coombs, 1990; Carpenter y Kirkland, 1998; Barrett, 2001), salvo en la terminología de los dentículos, para los que proponemos una nueva (Fig. 2F): al dentículo central lo denominados 0, y a partir de éste hacia el extremo mesial les damos un número correlativo (M1 ... Mn), y de la misma manera hacemos hacia el extremo distal (D1 ... Dn).

\section{SISTEMÁTICA}

Clase DINOSAURIA Owen, 1842

Orden ORNITHISCHIA Seeley, 1887

Nanorden THYREOPHORA Nopcsa, 1915

Suborden ANKYLOSAURIA Osborn, 1923

Familia Polacanthidae Wieland, 1911

Polacanthidae indet.

Figs. 2, 3 y 4, Tabla 3

Material: Dos dientes aislados depositados en el Museo Paleontológico de la Universidad de Zaragoza, siglados como MPZ 2002/926 y MPZ 2002/927 (este diente se fragmentó durante su preparación, por lo que no se ha podido fotografiar).

Descripción de MPZ 2002/926: es un diente mandibular izquierdo que conserva la corona completa y parte de la raíz, que está fracturada. Sus medidas se encuentran en la Tabla 3. Es un diente funcional al carecer de superficies de reabsorción y conserva parte de su raíz. La morfología general es «en forma de hoja», aunque menos desarrollada que en otros Ankylosauria. La longitud de la corona es mayor que la altura y la anchura es la dimensión menor (Tabla 3). El esmalte carece de ornamentación.

Se observa cómo la parte mesial de la base de la corona de

\begin{tabular}{|lcc|}
\hline & MPZ 2002/926 & MPZ 2002/927 \\
altura máxima conservada / maximun height & 5 & 1,9 \\
altura de la corona / crown height & 3,5 & 1,9 \\
longitud de la corona / crown length & 3,3 & 2,5 \\
anchura de la corona / crown width & 2,1 & 1,1 \\
altura de la raíz / root height & $>1,5(\mathrm{r})$ & $\mathrm{nc}$ \\
longitud de la raíz / root length & 2,2 & $\mathrm{nc}$ \\
anchura de la raíz / root width & 1,7 & $\mathrm{nc}$ \\
\hline
\end{tabular}

Tabla 3. Medidas en $\mathrm{mm}$ de los dientes de polacántido de La Cantalera, tránsito Hauteriviense-Barremiense (Josa, Teruel). Abreviaturas: nc: no conservada; r: roto. Tooth measurements (in mm) of the La Cantalera polacanthid, Hauterivian-Barremian transition (Josa, Teruel, Spain). Abbreviations: $n c$ : not preserved; $r$ : broken.

MPZ 2002/926 es más ancha que la distal. La corona y la raíz forman un ángulo aproximado de $120^{\circ}$ en el borde mesial, mientras que en el borde distal es aproximadamente de unos $150^{\circ}$. El lado labial es plano en sentido oclusal-adapical, mientras que el lingual es convexo. Carece de cíngulo en los dos lados, aunque en el lado labial presenta una base inflada, carácter que interpretamos como un cíngulo residual. Este hinchamiento es irregular, al tener un mayor desarrollo coincidiendo con el eje central del diente (Fig. 3).

Presenta un dentículo central (0), cuatro en el borde distal (D1 al D4) y cinco en el mesial (M1 al M5). Los dentículos son grandes, más largos apical-adapicalmente que mesiodistalmente. La separación entre ellos se prolonga ligeramente en cortos surcos en la corona. D1 y D2 están bien individualizados y tienen un tamaño similar, son puntiagudos con un terminación apical redondeada por el desgaste y más estrechos mesiodistalmente que el 0 . Su eje apical-adapical es prácticamente paralelo al dentículo $0\left(<20^{\circ}\right)$. El D3 está roto por su base. El D4 tiene un tamaño mucho menor, se sitúa en posición más labial que el resto y su terminación apical es más puntiaguda. Su eje apical-adapical se encuentra a unos $40^{\circ}$ del dentículo 0. En vista apical y distal se observa que los dentículos están unidos por una ligera carena mejor conservada en el área entre los dentículos, pero también en ellos.

En el borde mesial presenta cinco dentículos, pero el M4 y M5 prácticamente han sido erosionados por la faceta de desgaste que afecta a la parte mesio-labial del diente. La morfología de los dentículos es similar a la de los distales, pero están más desgastados por lo que la separación entre los dentículos está menos marcada. El M1 es casi paralelo al central, pero el M2 casi se presenta a $30^{\circ}$, el M3 a $40^{\circ}$, por lo que los dentículos mesiales se abren más en abanico que los distales. La transición entre raíz y corona es neta y está marcada por un cuello en el que el esmalte desaparece bruscamente (Fig. 3B). La sección de la raíz es subcircular, con la longitud algo mayor que la anchura y carece de cavidad pulpar. El esmalte es liso y aparentemente tiene similar desarrollo en ambos lados, con excepción de los dentículos, donde es más grueso (Fig. 3).

Como se ha comentado anteriormente, sobre la cara labial hay una faceta de desgaste prácticamente vertical que afecta al borde labio-distal. En las vistas labial y mesial se observa que 


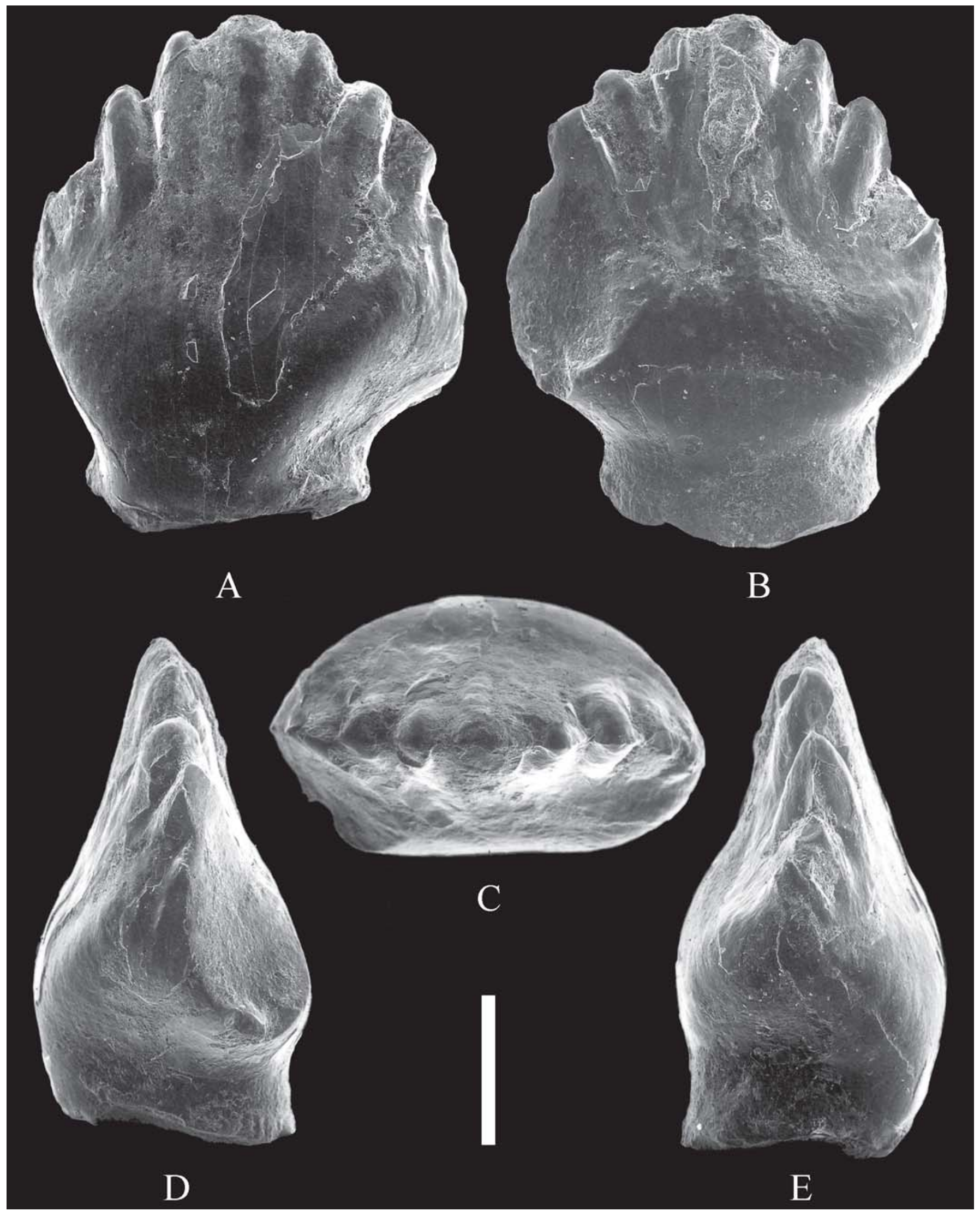

Figura 3. MPZ 2002/926. Diente mandibular izquierdo del polacántido de La Cantalera en vistas lingual (A), labial (B), oclusal (C), mesial (D) y distal (E). La escala es $1 \mathrm{~mm}$. MPZ 2002/926. Left dentary tooth of the La Cantalera polacanthid, in lingual (A), labial (B), occlusal (C), mesial (D) and distal (E) views. Scale bar $=1 \mathrm{~mm}$. 


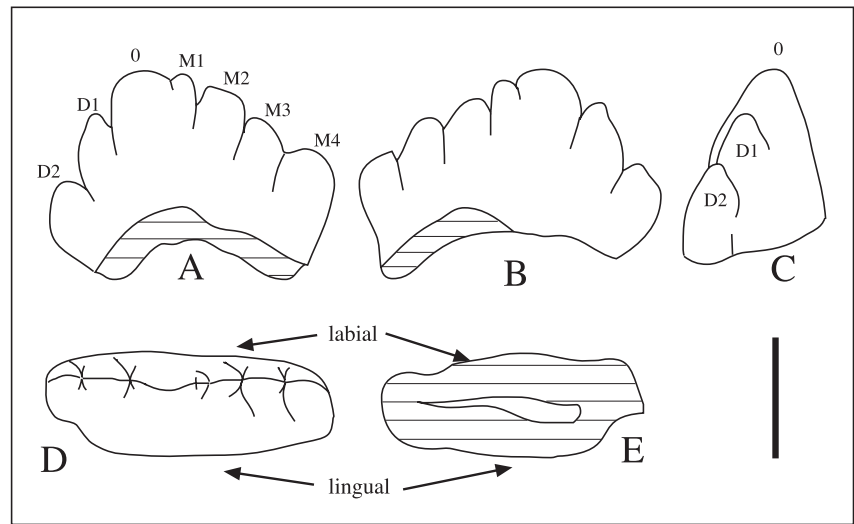

Figura 4. MPZ 2002/927. Germen dental mandibular izquierdo o maxilar derecho del polacántido de La Cantalera, en vistas lingual (A), labial (B), distal (C), oclusal (D) y adapical (E). Las líneas horizontales representan el esmalte, y la cavidad pulpar está en blanco. La escala es $1 \mathrm{~mm}$.

MPZ 2002/927. Left dentary or right maxillary germinal tooth of the La Cantalera polacanthid, in lingual (A), labial (B), distal (C), occlusal (D) and adapical (E) views. Horizontal lines represent the enamel, and the pulp cavity is in white. Scale bar = $1 \mathrm{~mm}$.

el borde de la faceta es denticulado, con un tamaño y forma similar a los dentículos del diente (Figs. 2 y 3). Además también poseen pequeñas facetas de desgaste el dentículo central y los situados en el borde mesial. Estas facetas están inclinadas hacía el lado labial. Son abundantes las estrías de alimentación que suelen tener dos direcciones preferentes, una subparalela a la mesio-distal y otra intermedia entre mesio-distal y labiolingual. Además en el diente se observan algunas marcas profundas distribuidas de manera aleatoria.

Descripción de MPZ 2002/927: es un diente mandibular izquierdo o maxilar derecho. Aflora la cavidad pulpar en su base y carece de facetas de desgaste, por lo que podría tratarse de un germen. La morfología general es similar a MPZ 2002/926, pero se diferencia por tener la base de la corona convexa y no cóncava como en MPZ 2002/926 y un número menor de dentículos. Hemos considerado que es el mismo taxón que MPZ 2002/926, estas diferencias pueden explicarse como la consecuencia de un desarrollo ontogenético incompleto o distinta posición dental.

MPZ 2002/927 tiene mayor longitud que altura, y ésta es mayor que la anchura (Fig. 4; Tabla 3). El lado labial es casi plano en vista oclusal-adapical, mientras que el lingual es convexo. La sección basal de MPZ 2002/927 es alargada, de forma lenticular, con los márgenes mesial y distal redondeados. El esmalte es liso, carece de ornamentación y aparentemente tiene el mismo desarrollo en el lado labial y en el lingual. Presenta un borde típicamente denticulado, el dentículo 0 es de mayor tamaño que el resto (M1 al M4 y D1 al D2). El 0 está distintivamente inclinado hacía el lado distal. Los dentículos están bien individualizados, y son más anchos que altos, pero más altos que largos. El M1 es más pequeño que el resto y está unido al dentículo 0. El resto tienen un tamaño similar. Están unidos por una carena en vista oclusal mejor desarrollada en la separación entre los dentículos.

\section{Discusión}

MPZ 2002/926 y 2002/927 tienen una morfología general en «forma de hoja» como en el resto de los anquilosaurios (Coombs y Maryanska, 1990), con la corona más larga (mesio-distalmente) que alta (apical-adapicalmente). Esta morfología tiene un cierto parecido a la de los dientes de estegosaurios; no obstante, según Barret (2001), los dientes laterales (maxilares y mandibulares) de los estegosaurios se diferencian de los de anquilosaurios, además de por su menor tamaño respecto al cráneo, por presentar dentículos redondeados, como por ejemplo en Hesperosaurus (Carpenter et al., 2001), mientras que en los anquilosaurios terminan en punta, por lo que MPZ 2002/926 y MPZ 2002/927 pueden identificarse como dientes de anquilosaurio.

La orientación de los dientes de Ankylosauria: La orientación de los dientes de Ankylosauria suele ser complicada, y cuando se encuentran aislados, los autores no suelen indicar su posición en la mandíbula (Coombs y Maryanska, 1990), o bien ésta no se discute (Kirkland, 1998). Sin embargo los dientes tienen suficientes elementos para poderlos orientar como se ha hecho en MPZ 2002/ 926 y MPZ 2002/927. En primer lugar se puede diferenciar el lado labial del lingual. En los dientes de Ankylosauria con cíngulo, como en los nodosáuridos, éste se encuentra más desarrollado en el lado labial (Coombs y Maryanska, 1990). En el caso de poseer un lado más inflado, como Pinacosaurus grangeri Gilmore, 1933, este lado también es el labial. MPZ 2002/926 tiene la base inflada en uno de los dos lados, que interpretamos como el labial. En vista apical el borde labial es prácticamente plano y el lingual es convexo; lo que nos ha permitido reconocer estos dos lados en el MPZ 2002/927.

Los dientes de Ankylosauria suelen ser ligeramente asimétricos y poseen un ligero desplazamiento del eje del diente hacía el margen distal (véanse las figuraciones de P. grangeri en Gilmore, 1933), como sucede en otros muchos dientes de dinosaurios. También se observa en Scelidosaurus donde los dientes están ligeramente inclinados distalmente (Barrett, 2001). Esto se traduce en que el borde mesial del diente tiene más dentículos y un ángulo mayor respecto al eje del diente. Utilizando este criterio se pueden reconocer los bordes mesial y distal en MPZ 2002/926: en vista lingual se observa que el diente no es simétrico, sino que uno de los lados está más desarrollado. Éste se interpreta como el mesial, y es precisamente en donde se encuentra la faceta de desgaste más importante y un mayor número de dentículos. En MPZ 2002/927 es más fácil diferenciar el lado mesial ya que tiene más dentículos y tiene mayor longitud. Incluso el dentículo 0 esta inclinado distalmente. 
Con los criterios discutidos hasta ahora, MPZ 2002/926 podría ser un diente mandibular izquierdo o un diente maxilar derecho, y para conocer cual es su posición podemos usar las facetas de desgaste. Éstas se forman por el contacto del diente contra el diente opuesto durante el procesado de los alimentos. En MPZ 2002/926, las facetas de desgaste se sitúan en el lado labial, tanto la que ha erosionado el borde mesial del diente como las que han desgastado los dentículos (Figs. 2 y 3). Los dientes maxilares de dinosaurio (y la mayoría de los vertebrados) tienen las facetas de desgaste en el lado lingual y los mandibulares en el labial (Barret, 2001); dado que las facetas de MPZ 2002/926 se encuentran en el lado labial este diente debe de ser un diente mandibular izquierdo.

La ausencia del cíngulo como carácter diagnóstico en Ankylosauria: La presencia/ausencia de cíngulo es el único carácter dental que suele utilizarse en las propuestas filogenéticas más modernas (Kirkland, 1998; Sereno, 1999; Carpenter, 2001; Vickaryous et al., 2001; Xu et al., 2001; Hill et al., 2003). Tradicionalmente se ha considerado que los nodosáuridos tienen un cíngulo conspicuo, mientras que los anquilosáuridos tienen un área inflada sin un cíngulo distintivo (Coombs, 1990; Coombs y Maryanska, 1990); sin embargo algunos de los dientes de Ankylosauridae figurados por estos autores, como Ankylosaurus y Euoplocephalus parecen presentar cíngulo. A este respecto, algunos autores consideran que estos dos géneros tienen cíngulo (Kirkland, 1998), otros que carecen de él (Vickaryous et al., 2001), o bien que Ankylosaurus no lo tiene pero Euoplocephalus sí lo tiene (Hill et al., 2003). Esta confusión se debe a la falta de caracterización del cíngulo: hay taxones como Minmi Molnar, 1996 que tienen dientes maxilares con un cíngulo bien delimitado formado por una banda que refuerza la base de la corona; sin embargo otros taxones presentan un cíngulo irregular, aunque también es una estructura bien delimitada del resto de la corona, como sucede en Euoplocephalus (ver Rybczynski y Vickaryous, 2001).

Si consideraramos el carácter «presencia de cíngulo» de manera amplia, la mayor parte de los Ankylosauria presentan cíngulo (Kirkland, 1998; Carpenter, 2001; Vickaryous et al., 2001; Xu et al., 2001). Sin embargo un abultamiento en la base de la corona no es un verdadero cíngulo (siguiendo a Kirkland, 1998), indudablemente se puede interpretar como el estadio intermedio entre cíngulo bien formado y ausencia de él (Pol et al., 1992), o bien un cíngulo residual. Se ha preferido restringir el término cíngulo a la presencia de una discontinuidad ligera o fuerte entre la base y el resto de la corona. El cíngulo verdadero puede coincidir con el denominado bandlike cingulum de Hill et al. (2003).

El siguiente paso es ponderar el peso de este carácter. Carpenter (2001) lista el carácter 14 de Ankylosauromorpha como cheek teeth with cingulum. Este nodo lo define como tireóforos más cercanos a Scelidosaurus, Minmi,
Polacanthidae, Nodosauridae y Ankylosauridae que a Stegosaurus, y por tanto equivale a los taxones que generalmente se han incluido en Ankylosauria + Scelidosaurus. En su matriz de caracteres considera que la presencia de cíngulo es el estadio derivado para Ankylosauromorpha. Esta interpretación es la que generalmente se ha seguido (Kirkland, 1998; Carpenter, 2001; Vickarious et al., 2001; Xu et al., 2001; Hill et al., 2003) a partir de Sereno (1984) que propone la diferenciación de un cíngulo como una sinapomorfia que comparten Stegosauria + Ankylosauria. Sin embargo la complejidad de este carácter la ponen de manifiesto Pol et al. (1992), apuntando que deberían considerarse los distintos estados de desarrollo, con lo cual estamos de acuerdo. Pero lo primero que debería discutirse es la polaridad de este carácter, y, como vamos a ver, consideramos que la ausencia de cíngulo es el estadio derivado. Esta es una consecuencia directa de la presencia en Scelidosaurus y otros taxones situados en nodos más primitivos, como Minmi (ver Carpenter, 2001) de un cíngulo bien desarrollado. Además todos los Nodosauridae, que tradicionalmente se han considerado como el grupo hermano de Ankylosauridae, tienen un cíngulo bien desarrollado (Coombs, 1990; Coombs y Maryanska, 1990), siendo algunos taxones derivados incluidos en Polacanthidae (o Polacanthinae) y Ankylosauridae los que carecen de él. Por tanto, teniendo en cuenta las propuestas filogenéticas actuales parece más adecuado considerar la ausencia de cíngulo como el estadio derivado.

Dientes de Ankylosauria sin cíngulo: Dado que interpretamos que el principal carácter diagnóstico del anquilosaurio de La Cantalera es la ausencia de cíngulo, vamos a revisar los Ankylosauria con este carácter. En el Jurásico se han citado tres taxones. Por una parte esta Gargoyleosaurus parkpini Carpenter, Miles y Cloward, 1998 descrito a partir de un cráneo de la Formación Morrison en Wyoming (EE.UU.). Los dientes de este taxón no han sido figurados, pero Carpenter et al. (1998) los describen como parecidos a los de ornitisquios primitivos, y en su listado de caracteres, el carácter 20 (presencia de cíngulo) para Gargoyleosaurus es 0, es decir sin cíngulo. Sarcolestes del Jurásico Medio (Formación Lower Oxford Clay) de Inglaterra, tiene dientes con la base inflada pero sin un verdadero cíngulo (Pereda-Suberbiola, 1993 contra Coombs y Maryanska, 1990).

En el yacimiento portugués de Pedrógao (Calloviense superior-Oxfordiense medio, véase discusión sobre la edad de este yacimiento en Ruiz-Omeñaca, 2001), Thulborn (1973) describe el taxón Alocodon kuehnei Thulborn, 1973 con dientes aislados. Inicialmente se incluyó en Fabrosauridae, sin embargo la peculiar morfología de estos dientes ha hecho que se los haya clasificado de diferentes maneras dentro de Ornithischia (Fabrosauridae, Hypsilophodontidae, Ornithischia incertae sedis, Thyreophora; véanse Ruiz-Omeñaca, 2001 y Knoll, 2002), así como que se haya considerado que el holotipo y el 
material referido puedan pertenecer a diferentes taxones (Ruiz-Omeñaca, 2001; Godefroit y Knoll, 2003). Independientemente de que se considere como un nomen dubium (Weishampel y Witmer, 1990; Knoll, 2002; Godefroit y Knoll, 2003), el holotipo (Thulbon, 1973; Galton, 1983) presenta caracteres que lo relacionan con los Thyreophora (corona simple, asimétrica y con dentículo apical grande; Galton, 1990) y con los Ankylosauria (dentículos con ápice afilado; Barret, 2001). El holotipo de Alocodon ha sido descrito como mandibular (Thulborn, 1973) y como maxilar izquierdo (Galton, 1983). Su atribución a un diente maxilar queda confirmada por el ligero desplazamiento distal del ápice y la posición lingual de las facetas de desgaste. Es un diente con forma palmeada, como otros Ankylosauria, con más longitud que altura. En la descripción original, Thulborn (1973) describe un cíngulo lingual en el holotipo de Alocodon; teniendo en cuenta que la cara lingual está fragmentada (Thulborn, 1973) no se aprecia bien si el cíngulo está bien desarrollado o es una base inflada. Un verdadero cíngulo sí está presente en algunos de los dientes aislados que incluye en este taxón (Thulborn, 1973). La morfología general del holotipo, incluyendo la forma, número y disposición de los dentículos, y la posible presencia de un cíngulo residual, es parecida a la de MPZ 2002/926. La única diferencia es la presencia en el lado lingual de ornamentación en el esmalte formada por finas crestas verticales (Galton, 1983), mientras que MPZ 2002/926 tiene el esmalte liso.

En el Cretácico Inferior de China se ha descrito recientemente Liaoningosaurus paradoxus Xu, Wang y You, 2001 con un ejemplar articulado del Barremiense de Liaoning (Formación Yixian). Xu et al. (2001) describen los dientes con cíngulo, sin embargo figuran un premaxilar y otro maxilar sin cíngulo. Esto deriva del problema anteriormente comentado sobre la interpretación de esta estructura. Independientemente de la presencia/ausencia de cíngulo, los dientes de Liaoningosaurus presentan unas crestas conspicuas a continuación de los dentículos que permiten diferenciarlo de MPZ 2002/926. Los dientes de Shamosaurus scutatus Tumanova, 1983, del Cretácico Inferior (Formación Hühteeg Svita) de Mongolia, no han sido descritos (Tumanova, 2000). Según Carpenter y Kirkland (1998) y Kirkland (1998; carácter 21, estadio 0) los dientes de este género carecen de cíngulo, aunque análisis posteriores (Xu et al., 2001, información suplementaria, carácter 44; Vickaryous et al., 2001, carácter 19; Hill et al., 2003, carácter 45) le dan un valor desconocido (?).

Un taxón interesante en esta discusión es Gastonia burgei Kirkland, 1998 procedente de la Formación Cedar Mountain (Barremiense) de Utah, EE.UU. (Kirkland, 1998). Se conoce tanto material craneal como postcraneal y un diente maxilar aislado. Se ha incluido en la familia Ankylosauridae, subfamilia Polacanthinae (Kirkland, 1998), o bien en la familia Polacanthidae (Carpenter,
2001). El diente aislado de Gastonia tiene una morfología similar a MPZ 2002/926, y únicamente se diferencia por poseer un mayor número de dentículos (16).

En el Cretácico Superior hay algunos ejemplos de taxones sin cíngulo incluidos en la familia Ankylosauridae, como el diente maxilar del holotipo de Pinacosaurus grangeri, ejemplar procedente de la Formación Djadochta en Mongolia (Gilmore, 1933). Este autor describe y figura un diente del holotipo que posee la base de la corona inflada en el lado labial, pero carece de un verdadero cíngulo. Se diferencia bien de MPZ 2002/926, porque en $P$. grangeri la separación entre los dentículos continúa en la superficie labial como surcos longitudinales, sin embargo en los dientes de La Cantalera los surcos están únicamente circunscritos a los dentículos. Hill et al. (2003) describen un cráneo juvenil de $P$. grangeri con dientes maxilares sin cíngulo (Hill et al., 2003), pero no describen los mandibulares. En la matriz de caracteres consideran a Pinacosaurus con cíngulo (Hill et al., 2003, carácter 45), quizá refiriéndose a que en su figura 8A los mandibulares parecen tener un cíngulo labial.

Como hemos visto la presencia/ausencia de cíngulo se considera un carácter importante en las filogenias modernas, pero hay diferencias en su interpretación en algunos taxones (Carpenter et al., 1998; Kirkland,1998; Vickaryous et al., 2001; Hill et al., 2003). Por ejemplo, Hill et al. (2003) consideran a Pawpawsaurus sin cíngulo, sin embargo según la descripción original de Lee (1996) los dientes maxilares tienen un cíngulo tanto en el lado labial, como en el lingual. Otro ejemplo es Scelidosaurus que presenta claramente un cíngulo (Barret, 2001; Carpenter, 2001), a pesar de que otros autores no lo han considerado como tal (Kirkland, 1998; Carpenter et al., 1998; Xu et al., 2001; Hill et al., 2003).

Con todo lo visto, puede decirse que sólo los «polacántidos», anquilosáuridos y algunos taxones de posición sistemática incierta como Alocodon carecen de cíngulo, mientras que todos los nodosaúridos lo presentan. Hay que destacar aquí que, erróneamente, Carpenter (2001: tabla 21.3) dice que Polacanthidae presenta cíngulo en los dientes laterales (carácter 14, estadio 1); sin embargo este autor incluye en Polacanthidae a Gargoyleosaurus, Gastonia, Hoplitosaurus, Hylaeosaurus, Mymmorapelta y Polacanthus, y sólo se conocen dientes de los dos primeros géneros, que, como ya se ha dicho, carecen de cíngulo.

Posición sistemática del anquilosaurio de La Cantalera: El esquema clásico de anquilosáuridos y nodosáuridos está cambiando en los últimos años por los descubrimientos en el Jurásico Superior y Cretácico Inferior de Norteamérica (Kirkland, 1998; Carpenter et al., 1998). La presencia de taxones con caracteres de anquilosáurido y de nodosáurido parece indicar que hay al menos otro grupo más, que Kirkland (1998) incluye en la subfamilia Polacanthinae teniendo en cuenta los caracteres craneales. Posteriores propuestas filogenéticas 
(Carpenter, 2001) elevan a nivel familiar (Polacanthidae) a estos anquilosaurios, que además de una armadura sacra osificada (siguiendo a Wieland, 1911), poseen otros caracteres diagnósticos. Aunque Kirkland (1998) y Carpenter (2001) reconocen como válida la familia Polacanthidae, otros autores (Vickaryous et al., 2001; Hill et $a l ., 2003)$ consideran que es parafilética.

El género Polacanthus está descrito en el Cretácico Inferior de la Isla de Wight (Inglaterra, Naish y Martill, 2001). El material tipo es fragmentario y la dentición es desconocida (Blows, 1987), aunque hay un diente aislado que se asigna a este género (Naish y Martill, 2001). Tradicionalmente este género se ha incluido en Nodosauridae (Blows, 1987; Pereda Suberbiola, 1994), siguiendo el esquema clásico. Autores como Pereda-Suberbiola (1994) han sugerido la sinonimia de Polacanthus con algunos taxones del Cretácico Inferior de Norteamérica como Hoplitosaurus. Esto ha sido confirmado por nuevos descubrimientos en la década de los 1990 que ha permitido conocer que en el Cretácico Inferior de Norteamérica y Europa había un grupo de Ankylosauria cercano a Polacanthus que incluía entre otros a Gastonia y Mymoorapelta (Kirkland y Carpenter, 1994; Kirkland, 1998; Carpenter, 2001). A partir de estos descubrimientos se han propuesto nuevas filogenias considerando a los «polacántidos» como un grupo diferenciado a nivel subfamiliar (Kirkland, 1998) o familiar (Carpenter, 2001). En este esquema se diferencian tres familias incluyendo en Polacanthidae a Polacanthus y a formas cercanas, como Gastonia del Cretácico Inferior de Norteamérica. Los Nodosauridae representan el grupo hermano del clado formado por Polacanthidae y Ankylosauridae (Carpenter, 2001). Existen otras propuestas filogenéticas como la de Vickaryous et al. (2001), en las que independientemente de considerar o no la anterior clasificación familiar, considera a los «polacántidos» de Carpenter (2001), como Gastonia o Gargoyleosaurus, como el grupo hermano del complejo grupo de los anquilosáuridos tradicionales (Ankylosauridae + Nodosauridae).

Hasta ahora, el único diente de anquilosaurio del Cretácico Inferior europeo descrito era uno asignado a Polacanthus del Weald inglés. Éste presenta un cíngulo discontinuo, un pequeño número de dentículos y una fuerte curvatura distal (Naish y Martill, 2001). El cíngulo lo diferencia de MPZ 2002/926 y de los polacántidos Gastonia y Gargoyleosaurus, siendo más parecido a los de Ankylosaurus (Coombs, 1990).

Con la propuesta filogenética de Carpenter (2001), los taxones que carecen de cíngulo se sitúan en las familias Polacanthidae y Ankylosauridae y por tanto hay que descartar que el Ankylosauria de la Cantalera sea un Nodosauridae. Esto estaría bastante de acuerdo con la propuesta clásica de nodosáuridos, los cuales tienen el cíngulo bien desarrollado (Coombs y Maryanska, 1990). La propuesta de Carpenter (2001) resulta interesante para nuestra dis- cusión porque los Ankylosauridae sin cíngulo como Pinacosaurus poseen una ornamentación en el esmalte que los diferencia de nuestro material. Sin embargo dientes como el de Gastonia incluido en Polacanthidae, son morfológicamente similares a los nuestros. Futuros estudios indicarán si la combinación de estos caracteres (ausencia de cíngulo y de ornamentación en el esmalte) son diagnósticos para los polacántidos, pero en el estado actual de conocimientos nos parece suficiente para incluir al Ankylosauria de La Cantalera en la familia Polacanthidae. En definitiva esta familia agrupa a una serie de taxones presentes exclusivamente en Norteamérica y Europa durante el Cretácico Inferior (Fig. 5).

\section{CONCLUSIONES}

Se estudian dos dientes de Ankylosauria del tránsito Hauteriviense-Barremiense de La Cantalera (Josa, Teruel). Sus principales características diagnósticas son la ausencia de cíngulo y el esmalte liso. La ausencia de cíngulo es un carácter derivado que presentan algunos anquilosáuridos, y que permite relacionarlo con Gastonia, un polacantino (o polacántido) del Barremiense de EE.UU. y con el holotipo de Alocodon kuehnei, un enigmático Ankylosauria del Jurásico Superior de Portugal. Dado lo fragmentario del material por el momento se considera al Ankylosauria de La Cantalera como un Polacanthidae indet. (o un Polacanthinae indet., según se considere a estos como subfamilia o familia).

Tradicionalmente se han incluido los restos de Ankylosauria del Cretácico Inferior de España en la familia Nodosauridae, algunos de los cuales se han relacionado con Polacanthus. El Ankylosauria de La Cantalera confirma la presencia de un taxón cercano a este género inglés, pero, dadas las nuevas propuestas filogenéticas, en el Hauteriviense-Barremiense de la Península Ibérica tenemos representado un taxon de la familia Ankylosauridae, la subfamilia Polacanthinae o la familia Polacanthidae, pero en cualquier caso separado de la familia Nodosauridae por la reducción o ausencia del cíngulo.

\section{AGRADECIMIENTOS}

El proyecto VECOBA esta financiado por el Ministerio de Ciencia y Tecnología (BTE2001-1746), la Fundación Conjunto Paleontológico de Teruel y el Gobierno de Aragón. Xabier Pereda-Suberbiola (Universidad del País Vasco) nos ha proporcionado varias referencia, incluido algún trabajo en prensa, y ha discutido con nosotros sobre la sistemática de los anquilosaurios y la validez de los dientes aislados. El Servicio de Microscopio Electrónica de la Universidad de Zaragoza ha realizado las fotografías. La empresa Paleoymás (Zaragoza) nos ha ayudado en las tareas de excavación del yacimiento de La 


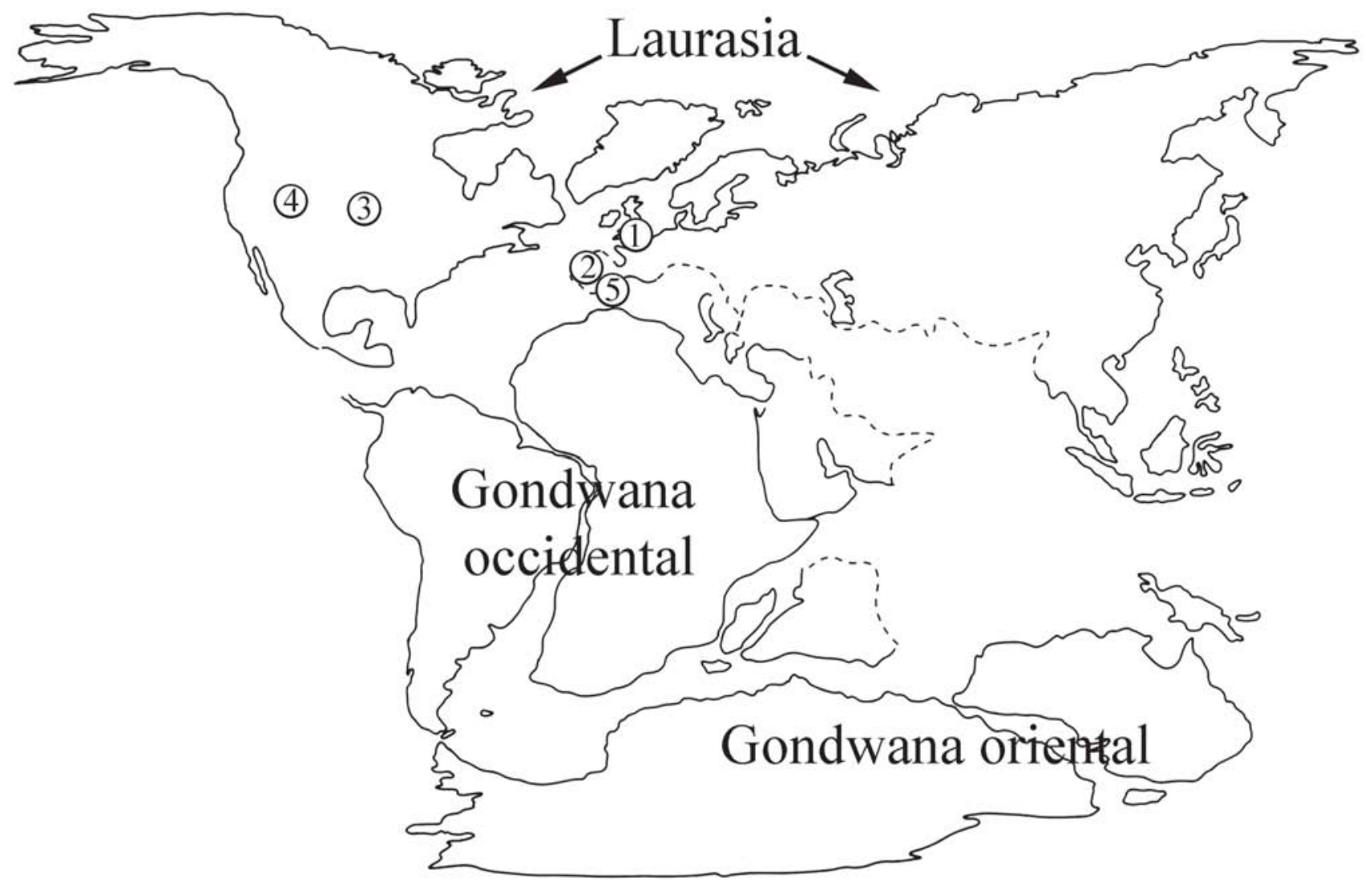

Figura 5. Reconstrucción paleogeográfica del Hauteriviense-Barremiense (modificado de Martill y Naish, 2001), con la situación de los polacántidos encontrados en estos pisos. 1: Polacanthus (Reino Unido), 2: Polacanthus (España), 3: Hoplitosaurus (= Polacanthus, South Dakota, EE.UU.), 4: Gastonia (Utah, EE.UU.), 5: Polacanthidae indet. (La Cantalera, España). Paleogeographical reconstruction of the Hauterivian-Barremian (after Martill y Naish, 2001). Labels show the situation of the polacanthids of this age. 1: Polacanthus (United Kingdom), 2: Polacanthus (Spain), 3: Hoplitosaurus (= Polacanthus, South Dakota, USA), 4: Gastonia (Utah, USA), 5: Polacanthidae indet. (La Cantalera, Spain).

Cantalera. Nuestro agradecimiento al Ayuntamiento de Josa, personalizado en su alcalde José Luis y la secretaría María José, por la ayuda constante en la excavación del yacimiento. Teresa, Alfredo, Amelia y Sylvia han procesado y triado parte de la muestra.

\section{REFERENCIAS}

Barrett, P. M. 2001. Tooth wear and possible jaw action of Scelidosaurus harrisonii Owen and a review of feeding mechanisms in other thyreophoran dinosaurs. In: The armored dinosaurs (Ed. K. Carpenter). Indiana University Press, Bloomington and Indianopolis, 25-52.

Blows, W. T. 1987. The armoured dinosaur Polacanthus foxii from the Lower Cretaceous of the Isle of Wight. Palaeontology, 30, 557-580.

Canudo, J. I., Amo, O., Cuenca-Bescós, G., Meléndez, A., Ruiz-Omeñaca, J.I. y Soria, A.R. 1997. Los vertebrados del Tithónico-Barremiense de Galve (Teruel, España). Cuadernos de Geología Ibérica, 23, 209-241.

Canudo, J. I., Aurell, M., Barco, J. L., Cobos, A., Cuenca-Bescós, G., Martín-Closas, C., Murelaga, X., Pereda-Suber- biola, X., Royo-Torres, R. y Ruiz-Omeñaca, J.I. 2002a. La Cantalera: Un resumen de los dinosaurios del tránsito Hauteriviense-Barremiense (Cretácico inferior) de la Cordillera Ibérica (Josa, Teruel). In: Libro de Resúmenes. XVIII Jornadas de la Sociedad Española de Paleontología. II Congreso Ibérico de Paleontología, Salamanca, 27-28.

Canudo, J. I., Cuenca-Bescós, G., Ruiz-Omeñaca, J. I., Soria, A. R. 1996. Registro fósil de vertebrados en el tránsito Jurásico-Cretácico de Galve (Teruel, España). Revista de la Academia de Ciencias Exactas, Físicas, Químicas y Naturales de Zaragoza, Serie $2^{a}$, 51, 221-236.

Canudo, J. I., Ruiz-Omeñaca, J. I., Barco, J. L. y Royo-Torres, R. 2002b. ¿Saurópodos asiáticos en el Barremiense inferior (Cretácico Inferior) de España? Ameghiniana, 39, 443-452.

Carpenter, K. 2001. Phylogenetic analysis of the Ankylosauria. In: The armored dinosaurs (Ed. K. Carpenter). Indiana University Press, Bloomington and Indianopolis, 455483.

Carpenter, K. and Kirkland, J. I. 1998. Review of Lower and Middle Cretaceous Ankylosaurs from North America. New Mexico Museum of Natural History and Science Bulletin, 14, 249-270. 
Carpenter, K., Miles, C. A. and Cloward, K. 1998. Skull of a Jurassic ankylosaur (Dinosauria). Nature, 393, 782-783.

Carpenter, K., Miles, C. A. and Cloward, K. 2001. New Primitive Stegosaur from the Morrison Formation, Wyoming. In: The armored dinosaurs (Ed. K. Carpenter). Indiana University Press, Bloomington and Indianopolis, 55-75.

Coombs Jr., W. P. 1978. The families of the Ornitischian dinosaur order Ankylosauria. Palaeontology, 21, 143-170.

Coombs Jr., W. P. 1990. Teeth and taxonomy in ankilosaurs. In: Dinosaurs systematics. Approaches and Perspectives (Eds. K. Carpenter and P. J. Currie). Cambridge University Press, Cambridge, 269-280.

Coombs, W. P. and Maryanska, T. 1990. Ankylosauria. In: The Dinosauria (Eds. D. B. Weishampel, P. Dodson and H. Osmólska). University of California Press, Berkeley, Los Angeles, Oxford, 456- 483.

Díaz-Molina, M., Yébenes, A., Goy, A. et Sanz, J. L. 1985. Le Jurassique Superieur detritique-carbonaté du synclinal de Galve (Teruel). Strata, Série 2, 2, 155-182.

Fuentes Vidarte, C., Meijide Calvo, M., Meijide Fuentes, F., Meijide Fuentes, M. y Sanz Aldea, F. 2002. Primeros restos de Iguanodon (Dinosauria, Ornithischia) en el Cretácico Inferior de Soria (España). In: Congreso Internacional sobre dinosaurios y otros reptiles mesozoicos de España (Ed. F. Pérez-Lorente), Logroño, 17.

Fuentes Vidarte, C., Meijide Calvo, M., Meijide Fuentes, M., Meijide Fuentes, F. 2003. Fauna mesozoica del yacimiento de «Zorralbo» en Golmayo (Soria, España). In: Libro de Resúmenes de las XIX Jornadas de Paleontología. Morella 2003 (Eds. M. V. Pardo Alonso y R. Gozalo), Morella, 76.

Galton, P. M. 1983. The cranial anatomy of Dryosaurus, a Hypsilophodontid dinosaur from the Upper Jurassic of North America and East Africa, whit a review of hypsilophodontids from the Upper Jurassic of North America. Geologica et Palaeontologica, 17, 207-243.

Galton, P. M. 1990. Stegosauria. In: The Dinosauria (Eds. D. B. Weishampel, P. Dodson and H. Osmólska). University of California Press, Berkeley, Los Angeles, Oxford, 435-455.

Gasulla, J. M., Ortega, F., Pereda Suberbiola, X. y Sanz, J. L. 2003. Elementos de la armadura dérmica del dinosaurio anquilosaurio Polacanthus (Cretácico Inferior. Morella, castellón, España). In: Libro de Resúmenes de las XIX Jornadas de Paleontología. Morella 2003 (Eds. M. V. Pardo Alonso y R. Gozalo), Morella, 83.

Gilmore, C. W. 1933. Two new dinosaurian reptiles from Mongolia with notes on some fragmentary specimens. American Museum Novitates, 679, 1-20.

Godefroit, P. and Knoll, F. 2003. Late Triassic dinosaur teeth from southern Belgium. Comptes Rendus Palevol, 2, 3-11.

Hill, R. V., Witmer, L. M. and Norell, M. A. 2003. A new specimen of Pinacosaurus grangeri (Dinosauria: Ornithischia) from the Late Cretaceous of Mongolia: Ontogeny and phylogeny of ankylosaurs. American Museum Novitates, 3395, 1-29.
Kirkland, J. I. 1998. A Polacanthine ankylosaur (Ornithischia: Dinosauria) from the Early Cretaceous (Barremian) of eastern Utah. New Mexico Museum of Natural History and Science Bulletin, 14, 270-281.

Kirkland, J. I. and Carpenter, K. 1994. North America's First Pre-Cretaceous Ankylosaur (Dinosauria) from the Upper Jurassic Morrison Formation of Western Colorado. Brigham Young University Geology Studies, 40, 25-42.

Knoll, F. 2002. Les Fabrosauridae Galton, 1972 (Dinosauria: Ornithischia): répartition géographique et stratigraphique; systématique et phylogénie. Tesis Doctoral, Muséum national d'Histoire naturelle, Paris,1-242 (inédita).

Lee, Y. N. 1996. A new nodosaurid ankylosaur (Dinosauria: Ornithischia) from the Paw Paw Formation (Late Albian) of Texas. Journal of Vertebrate Paleontology, $\mathbf{1 6}$, 232-245.

Martill, D. M. and Naish, D. 2001. The global significance of the Isle of Wight dinosaurs. In: Dinosaurs of the Isle of Wight (Eds. D. M. Martill and D. Naish). The Paleontological Association, London, 44-48.

Martín-Closas, C. 2000. Els caròfits del Juràssic superior $i$ el Cretaci inferior de la Península Ibérica. Institut d'estudis catalans, Barcelona,1-304.

Martín-Closas, C. y Alonso-Millán, A. 1998. Estratigrafía y bioestratigrafía (Charophyta) del Cretácico inferior en el sector occidental de la Cuenca de Cameros (Cordillera Ibérica). Revista de la Sociedad Geológica de España, 11, 253-269.

McCrea, R., Lockley, M. G. and Meyer, C. A. 2001. Global distribution of purported ankylosaur track occurrences. In: The armored dinosaurs (Ed. K. Carpenter). Indiana University Press, Bloomington and Indianopolis, 413-454.

Molnar, R. E. 1996. Preliminary report on a new ankylosaur from the Early Cretaceous of Queensland, Australia. Memoirs of the Queensland Museum, 39, 653-668.

Naish, D. and Martill, D. M. 2001. Armoured dinosaurs: thyreophorans. In: Dinosaurs of the Isle of Wight (Eds. D. M. Martill and D. Naish). The Paleontological Association, London, 147-184.

Nopcsa, F. 1915. Die Dinosaurier der siebengürgischen Landesteile Ungarns. Mitteilungen aus dem Jahrbuch der Ungarischen Geologischen Reichsanstalt, 23, 1-26.

Osborn, H. F. 1923. Two Lower Cretaceous dinosaurs from Mongolia. American Museum Novitates, 95, 1-10.

Owen, R. 1842. Report on British fossil reptiles. Part II. Report of the British Association of the Advancement of Science, 11, 60-204.

Pereda-Suberbiola, X. 1993. Les dinosaures ankylosauriens d'Europe: systématique et evolution. Mémoires des Sciences de la Terre Université Curie, Paris, 9320, 1-302.

Pereda-Suberbiola, J. 1994. Polacanthus (Ornithischia, Ankylosauria), a transatlantic armoured dinosaur from the Early Cretaceous of Europe and North America. Palaeontographica. Abteilung A: Palaeozoologie-Stratigraphie, 232, 4-6.

Pereda Suberbiola, X. and Galton, P. M. 2001. Thyreophoran ornithischian dinosaurs from the iberian peninsula. In: Actas de las I Jornadas internacionales sobre paleontolo- 
gía de dinosaurios y su entorno. (Ed. Colectivo Arqueológico-Paleontológico de Salas). Salas de los Infantes, 147-161.

Pereda Suberbiola, X., Galton, P. M., Torcida, F., Huerta, P., Izquierdo, L.A., Montero, D., Pérez, F. and Urién, V. 2003. First stegosaurian dinosaur remains from the Early Cretaceous of Burgos (Spain). Revista Española de Paleontología, 18, 143-150.

Pereda Suberbiola, X., Meijilde, M., Torcida, F., Welle, J., Fuentes, C., Izquierdo, L.A., Montero, D., Pérez, F. y Urién, V. 1999. Espinas dérmicas del dinosaurio anquilosaurio Polacanthus en las facies Weald de Salas de los Infantes (Burgos, España). Estudios geológicos, 55, 267-272.

Pol, C., Buscalioni, A. D., Carballeira, J., Francés, V., López Martínez, N., Marandat, B., Moratalla, J. J., Sanz, J. L., Sigé, B. y Villatte, J. 1992. Reptiles and mammals from the Late Cretaceous new locality Quintanilla del Coco (Burgos Province, Spain). Neues Jahrbuch für Geologie und Paläontologie, Abhandlungen, 184, 279-314.

Ruiz-Omeñaca, J. I., 2001. Dinosaurios hipsilofodóntidos (Ornithischia: Ornithopoda) en la Península Ibérica. In: Actas de las I Jornadas internacionales sobre paleontología de dinosaurios y su entorno (Ed. Colectivo Arqueológico-Paleontológico de Salas). Salas de los Infantes, $175-266$.

Ruiz-Omeñaca, J. I. y Canudo, J. I. 2001. Vallipón y La Cantalera: dos yacimientos paleontológicos excepcionales. Naturaleza Aragonesa, 8, 8-17.

Ruiz-Omeñaca, J. I. y Canudo, J. I. 2003. Un nuevo dinosaurio terópodo ("Prodeinodon" sp.) en el Cretácico Inferior de La Cantalera (Teruel). Geogaceta, 34, 111-114.

Ruiz-Omeñaca, J. I. y Canudo, J. I. en prensa. Dinosaurios (Saurischia, Ornithischia) en el Barremiense (Cretácico Inferior) de la Península Ibérica. Ciencias de la Tierra.

Ruiz-Omeñaca, J. I., Canudo, J.I. y Cuenca-Bescós, G. 1997. Primera evidencia de un área de alimentación de dinosaurios herbívoros en el Cretácico Inferior de España (Teruel). Monografías de la Academia de Ciencias Exactas, Fisicas, Químicas y Naturales de Zaragoza, Serie 2. , 10,1-48.

Rybczynski, N. and Vickaryous, M. K. 2001. Evidence of complex jaw movements in the Late Cretaceous ankylosaurid Euoplocephalus tutus (Dinosauria: Thyreophora). In: The armored dinosaurs (Ed. K. Carpenter). Indiana University Press, Bloomington and Indianopolis, 299-317.

Santafé, J. V., Casanovas, M. L., Sanz, J. L. y Calzada, S. 1982. Geología y paleontología (Dinosaurios) de las Capas Rojas de Morella (Castellón, España). Diputación Provincial de Castellón y Diputación de Barcelona, Castellón y Barcelona, 1-169.

Sanz, J. L. 1983. A nodosaurid ankylosaur from the Lower Cretaceous of Salas de los Infantes (Province of Burgos, Spain). Géobios, 16, 615-621.

Sanz, J. L., Buscalioni, A. D., Casanovas, M. L. y Santafé, J. V. 1987. Dinosaurios del Cretácico Inferior de Galve (Teruel, España). Estudios geológicos, volumen extraordinario Galve-Tremp, 45-64.
Sanz, J. L., Buscalioni, A. D., Moratalla, J. J., Francés, V. y Antón, M. 1990. Los reptiles mesozoicos del registro español. Monografías del Museo Nacional de Ciencias Naturales, 2, 1-79.

Sanz, J. L., Buscalioni, A. D., Pérez-Moreno, B., Moratalla, J. y Jiménez García, S., 1992. Los dinosaurios de Castilla y León. In: Vertebrados fósiles de Castilla y León (Ed. E. Jiménez-Fuentes ). Museo de Salamanca, Salamanca, 47-57.

Seeley, H. G.1887. On the classification of the fossil animals commonly called Dinosauria. Proceedings of the Royal Society London, 43, 165-171.

Sereno, P. C.1984. The phylogeny of the Ornithischia: A reappraisal. In: Third Symposium on Mesozoic Terrestrial Ecosystem Short Papers (Ed. W. E. Reif and F. Westphal). Attempto Verlag, Tübingen, 219-226.

Sereno, P. C. 1999. A rationale for dinosaurian taxonomy. Journal of Vertebrate Paleontology, 19, 788-790.

Soria de Miguel, A. R. 1997. La sedimentación en las cuencas marginales del Surco Ibérico durante el Cretácico Inferior y su contorno estructural. Servicio de Publicaciones de la Universidad de Zaragoza, Zaragoza,1-363.

Thulborn, R. A. 1973. Teeth of ornithischian dinosaurs from the Upper Jurassic of Portugal. Memorias Serviços Geológicos de Portugal (nova série), 22, 89-134.

Torcida Fernández-Baldor, F. 1996. Registro de dinosaurios en el sureste de la provincia de Burgos. Zubía, 14, 89-104.

Torcida Fernández-Baldor, F. Izquierdo Montero, L. A., Huerta Hurtado, P., Montero Huerta, D., Pérez Martínez, G. En prensa. Dientes de dinosaurios (Theropoda, Sauropoda) en el Cretácico Inferior de Burgos (España). Ciencias de la Tierra.

Tumanova, T. A. 1983. El primer anquilosaurio del Cretácico Inferior de Rusia. (en ruso). Trudy Sovmestnoi SovetskoMongol'skoi Paleontologicheskoi Expeditsii, 24, 110-120.

Tumanova, T. A. 2000. Armoured dinosaurs from the Cretaceous of Mongolia. In: The Age of Dinosaurs in Russia and Mongolia (Eds. M. J. Benton, M. A. Shishkin, D. M. Unwin and E. N. Kurochkin). Cambridge University Press, Cambridge, 517-532.

Vickaryous M. K., Russell A. P., Currie P. J. and Zhao X.-J. 2001. A new ankylosaurid (Dinosauria: Ankylosauria) from the Lower Cretaceous of China, with comments on ankylosaurian relationships. Canadian Journal of Earth Sciences, 38, 1767-1780.

Weishampel, D. B. and Witmer, L. M. 1990. Lesothosaurus, Pisanosaurus, and Technosaurus. In: The Dinosauria (Eds. D. B. Weishampel, P. Dodson and H. Osmólska). University of California Press, Berkeley, Los Angeles, Oxford, 416-425.

Wieland, G. 1911. Notes of the armored Dinosauria. American Journal of Science, (Series 4), 31, 112-124.

Xu, X., Wang, X-L. and You, H.-L. 2001. A juvenile ankylosaur from China. Naturwissenschaften, 88, 297-300.

Manuscrito recibido: 22 de mayo, 2003 Manuscrito aceptado: 19 de noviembre, 2003 